\title{
i-Car: An Intelligent and Interactive Interface for Driver Assistance System
}

\author{
Hemanth Kumar G*, Zarif Ahmed, Anusha Shetty, Nissar and Vineeth Bangera \\ Department of Computer Science, NMAM Institute of Technology, Karkala Taluk, Udupi District, Karnataka \\ 574110, State Highway 1, Nitte, Karnataka 574110, India
}

\begin{abstract}
Article Information

The aim of the present research was to reduce accidents by assisting the driver in various aspects of driving such as lane detection, pedestrian and car detection, driver drowsiness detection and rear view parking assistance. The methodology combines the computer vision techniques with pattern recognition, feature extraction, machine learning, object recognition, human computer interaction and parallel processing in a nutshell. The proposed system provides robust extraction of lane markings in various types and alerts the driver attempting to drift from the lane. It also detects the pedestrians and cars which are at a vulnerable distance to be hit by the vehicle and alarms the driver well ahead of time. The system uses eye closure based decision algorithm to detect driver drowsiness in all conditions and also warns by interactive voice early enough to avoid the accidents. It also assists the driver while reversing the vehicle, by providing a clear view of his blind spot areas. Computer vision algorithms like Hough's Transform, Canny Edge detection and HAAR classifiers were applied to meet the objectives. The integrated module was analyzed and tested in different terrains and various lighting condition to produce an accurate and robust real-time assistance system (Sivaraman et al., 2014). iCar is an innovative prototype in the Information Technology with minimum hardware like low cost webcams. It emerged as an Interactive Technology with an interactive audio, visual, touch and touch-less interfaces. These can assist to avoid accidents in the world by intelligently ignoring certain hardware sensors like IR, UV, Acoustic, Proximity and mechanical devices like costlier LIDAR (Light Detection and Ranging) fitted in Google Car. Present research findings outperform the state of the art research like CalTech (Aly et al., 1997). Attempts of depth sensing even using Microsoft Kinect could be ignored by the present technology, the iCar.

Article History:

Received : 20-02-2014

Revised : 25-05-2014

Accepted : 28-05-2014

Keywords:

iCar

Canny Edge detection

HAAR Classifier

Probabilistic Hough's

Transform

*Corresponding Author:

Hemanth Kumar G

E-mail:

hemanthjois@gmail.com Copyright@2014 STAR Journal. All Rights Reserved.
\end{abstract}

\section{INTRODUCTION}

According to the road traffic injuries fact sheet $\left(\mathrm{N}^{\circ} 358\right)$ published by World Health Organization on March 2013, every year the lives of almost 1.24 million people are cut short as a result of a road traffic crash. Around 20 to 50 million more people suffer non-fatal injuries, with many incurring a disability as a result of their injury. Road traffic injuries cause considerable economic losses to victims, their families, and to nations as a whole. These losses arise from the cost of treatment (including rehabilitation and incident investigation) as well as reduced/lost productivity (e.g. in wages) for those killed or disabled by their injuries, and for family members who need to take time off work (or school) to care for the injured (Ministry of Road Transport and Highways of India, Transport Research Wing, 2012).

There are few global estimates of the costs of injury, but an estimate carried out in 2000 suggest that the economic cost of road traffic crashes was approximately US\$ 518 billion. National estimates have illustrated that road traffic crashes cost countries between 1-3\% of their gross national product, while the financial impact on individual families has been shown to result in increased financial borrowing and debt, and even a decline in food consumption. Road traffic injuries have been neglected from the global health agenda for many years, despite being predictable and largely preventable. Evidence from many countries shows that dramatic successes in preventing road traffic crashes can be achieved through concerted efforts that involve, but are not limited to, the health sector.

\section{MATERIALS AND METHODS}

Figure 1 illustrates the brief methods were used in iCar. The description of all the methods were as follows:

\section{Lane Detection}

Video Acquisition: Here camera was mounted on the vehicle which was capable of reaching real time performances in detection and tracking of structured road boundaries (Painted or Unpainted Lane markings) with slight curvature, which was robust enough in presence of shadow and other worst case scenarios.

Frame Extraction: The video acquired was given as an input, from which individual frames were extracted.

Image Processing: This step involved preparing the frames for the next step. The image was first given as 
input to a Gaussian blur algorithm which gave a blurred output so that only well-defined edges are detected. Then the image was also converted into grayscale to increase efficiency of edge detection.

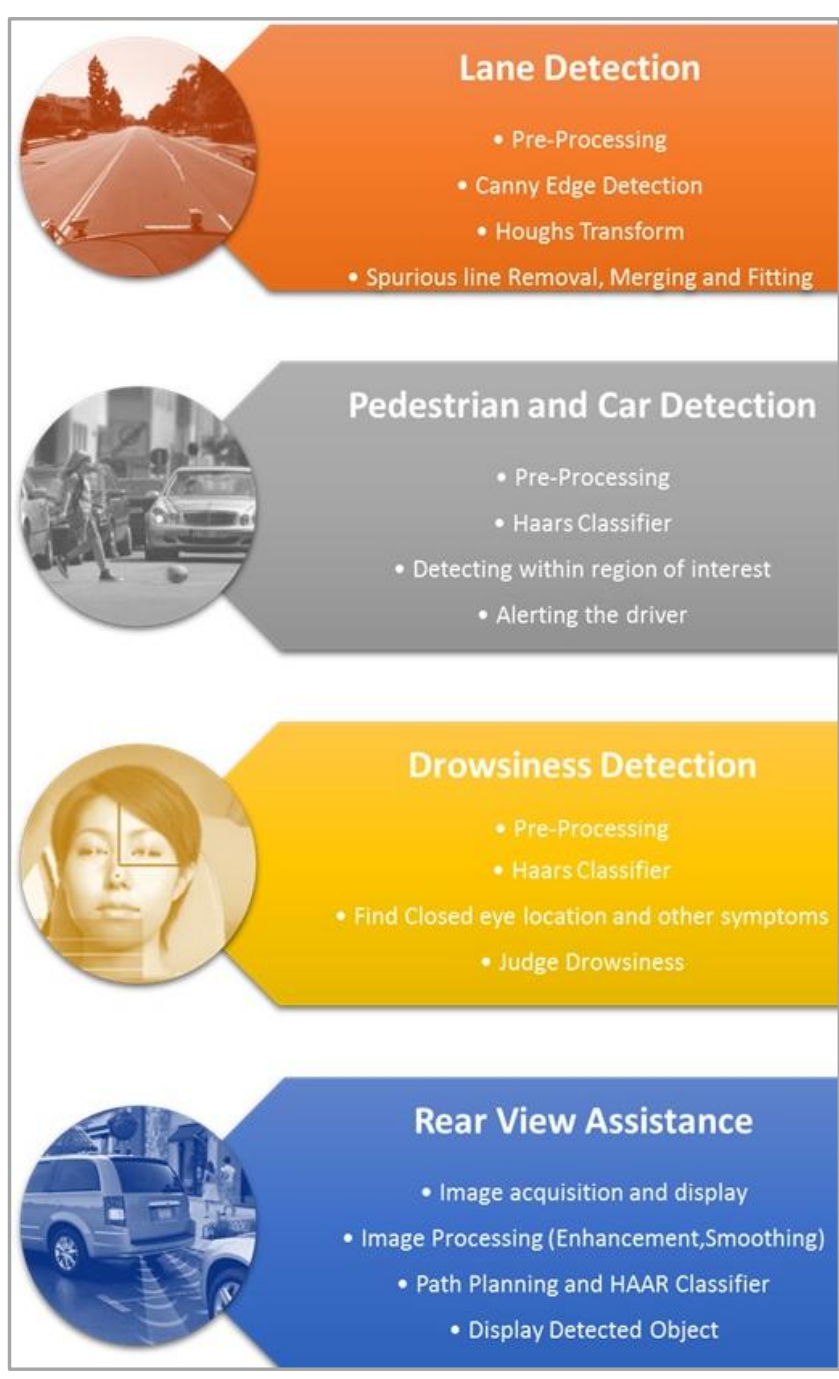

Figure 1: Different methods used in i-Car.

Canny Edge Detection: This algorithm helped in identifying all the edges of the image.

Hough's Transform: A technique to detect arbitrary shapes in images, given a parameterized description of the shape in question. Hough transform (Hillel et al., 2014) can detect imperfect instances of the searched shapes. Besides, it is tolerant of gaps, and image noise has minor effect on the output (Chen et al., 2014).

Spurious Line Removal and Line Merging: The spurious lines were removed and merging of lines is done by grouping them according to their similarity in slope and spatial closeness. It is done, based on the following:

i. The lane markings should be somewhat parallel to each other and they should merge at vanishing Point.

ii. Starting point of the left and right lane mark should be in $3^{\text {rd }}$ and $4^{\text {th }}$ quadrant respectively for a given image divided into four quadrants.

iii. The slope of the line in the near sight should be approximately vertical and continuous in the latter part.

\section{Pedestrian and Car Detection}

Specifying Region of Interest: i-Car should be concerned only about those pedestrians who are crossing the road, thus the region of interest will be the road in the frame. Only that part of the frame was extracted for further processing.

HAAR Classifier: It is a data file generated after a training process. It contains descriptors about the object which are to be detected. In this case the Pedestrians (Prioletti et al., 2013) and the Cars. A classifier was trained with a few hundred sample views of a particular object (i.e., a face or a car), called positive examples, that are scaled to the same size (say, 20x20), and negative examples - arbitrary images of the same size. Once the classifier is trained, it was applied to a region of interest (of the same size as used during the training) in an input image. To search for the object in the whole image, one can move the search window across the image and check every location using the classifier. The classifier is designed so that it can be easily "resized" in order to be able to find the objects of interest at different sizes, which is more efficient than resizing the image itself. So, to find an object of an unknown size in the image, the scan procedure should be done several times at different scales (Viola et al., 2003).

\section{Driver Drowsiness Detection}

Tracking the Eye and Displaying the Findings: When there are 5 consecutive frames find the closed eye scenario, an alarm is activated, and the driver is alerted to wake up (Rezaee et al., 2013). Consecutive number of closed frames is needed to avoid including instances of eye closure due to blinking.

Path Planning: The path was divided into 3 blocks consisting red, yellow and green color. These blocks were used to guide the driver by indicating whether it was safe or whether he needs to slow down or stop.

Warning: If the detected object lies on the danger zone (i.e. red block), an alert will be generated to the driver by a strong message. If the detected object lies on the yellow block, warn the driver by using an audio beep.

\section{RESULTS AND DISCUSSION}

Driver Drowsiness Detection: The program when executed to detect the open eyes, gave accurate results but when the program was tried to detect the closed eyes gave inappropriate results. After thorough analysis, it was found that the HAAR training to detect closed eyes wasn't done properly (Garcia et al., 2012). The solution to this was generating an accurate $\mathrm{xml}$ file which was used in the program. Instead of using 600 positive images and equal number of negative images, the HAAR training was done by using the positive and negative images in the ratio $2: 3$ and taking about 1000 positive images. The detection rate was $87.35 \%$. The performance of this module is dependent on different driver poses, orientations, lighting conditions and low cost camera limitations.

Lane Detection: The individual module, lane detection could detect the lane in real time and didn't give any lag but when integrated with pedestrian detection module the time lag was very high and hence very inefficient. The processing speed of the module was increased by parallel processing. The frames extracted were used to detect the 
Hemanth Kumar et al.,

individual objects like pedestrian and cars but while sending the output to the interface, it was combined and the results were displayed together. The program is robust to detect the lanes during night and also during extreme weather conditions like rain or snow (Qingquan $\mathrm{Li}$ et al., 2014). The canny edge detection algorithm detects these edges based on some threshold value. For e.g. the threshold value for cloudy weather will be lesser compared to normal sunny day. Hence the threshold is set in such a way that it detects almost all necessary edges in an accurate way under any condition.

Main module Lane Detection was analyzed by collecting the parameters of confusion matrix. Table 1
Sci. Technol. Arts Res. J., April-June 2014, 3(2): 197-200

shows the Dataset used and actual number of Lane Boundaries found out by manual annotation. Table 2 shows that the Type-I and Type-II error rates are 6.86\% and $4.96 \%$ respectively. The performance evaluation done for hypothesis testing from Table 2 depicts that Positives are more than negative alarm. Table 3 proves that the lane detection module is $92.44 \%$ Sensitive to reject improper lanes and in $71.55 \%$ specificity, it could find lanes both in Urban and rural scenarios. Table 4 shows that the Accuracy of the system is $82.7 \%$. This is due to tradeoff between the Machine learning algorithms and low cost devices used in embedded computer vision programming constraints.

Table 1: Real Time Video Clip Dataset for Lane Detection

\begin{tabular}{cccc}
\hline Clip & Name & Frames & Lane Boundaries \\
\hline 1 & Sample 1 & 280 & 906 \\
2 & Sample 2 & 450 & 998 \\
3 & Sample 3 & 370 & 1074 \\
4 & Sample 4 & 200 & 912 \\
\hline Total & & $\mathbf{1 3 0 0}$ & $\mathbf{3 8 9 0}$ \\
\hline
\end{tabular}

Table 2: Positive and Negative Predictive Values for Lane Detection

\begin{tabular}{ccccccc}
\hline Clip & $\begin{array}{c}\text { Lane } \\
\text { Boundaries }\end{array}$ & $\begin{array}{c}\text { Lanes } \\
\text { Detected }\end{array}$ & $\begin{array}{c}\text { True } \\
\text { Positive }^{*}\end{array}$ & $\begin{array}{c}\text { True } \\
\text { Negative }^{*}\end{array}$ & $\begin{array}{c}\text { False } \\
\text { Positive }^{*}\end{array}$ & $\begin{array}{c}\text { False } \\
\text { Negative }^{*}\end{array}$ \\
\hline 1 & 906 & 780 & 565 & 112 & 63 & 40 \\
2 & 998 & 806 & 583 & 156 & 39 & 28 \\
3 & 1074 & 998 & 612 & 214 & 98 & 74 \\
4 & 912 & 873 & 568 & 187 & 67 & 51 \\
\hline Total & $\mathbf{3 8 9 0}$ & $\mathbf{3 4 5 7}$ & $\mathbf{2 3 2 8}$ & $\mathbf{6 6 9}$ & $\mathbf{2 6 7}$ & $\mathbf{1 9 3}$
\end{tabular}

*True Positive: Lane correctly recognized as lane; *False Positive: Non-lane incorrectly recognized as lane;

${ }^{*}$ False Negative: Lane incorrectly rejected as Non-lane and *True Negative: Non-lane correctly rejected as Non-lane

Table 3: Evaluation of Lane Detection classifier performance

\begin{tabular}{ccccc}
\hline Clip & $\begin{array}{c}\text { Sensitivity } \\
(\%)\end{array}$ & $\begin{array}{c}\text { Specificity } \\
(\%)\end{array}$ & $\begin{array}{c}\text { Precision } \\
(\%)\end{array}$ & $\begin{array}{c}\text { Negative Predictive } \\
\text { Value (\%) }\end{array}$ \\
\hline 1 & 93.38 & 64 & 89.96 & 73.68 \\
2 & 95.41 & 80 & 93.72 & 84.78 \\
3 & 89.21 & 68.58 & 86.19 & 74.3 \\
4 & 91.76 & 73.62 & 89.44 & 78.57 \\
\hline Average (\%) & $\mathbf{9 2 . 4 4}$ & $\mathbf{7 1 . 5 5}$ & $\mathbf{8 9 . 8 3}$ & $\mathbf{7 7 . 8 3}$ \\
\hline
\end{tabular}

Table 4: Parameters of Contingency Table for Lane Detection

\begin{tabular}{ccccc}
\hline Clip & $\begin{array}{c}\text { Fall-out } \\
(\%)\end{array}$ & $\begin{array}{c}\text { False Discovery } \\
\text { Rate (\%) }\end{array}$ & $\begin{array}{c}\text { Accuracy } \\
(\%)\end{array}$ & $\begin{array}{c}\text { Harmonic mean of } \\
\text { precision and } \\
\text { sensitivity (\%) }\end{array}$ \\
\hline 1 & 36 & 10 & 84 & 91.64 \\
2 & 20 & 6 & 74 & 94.56 \\
3 & 31.4 & 13 & 76.9 & 87.67 \\
4 & 26.3 & 9 & 82.7 & 90.59 \\
\hline
\end{tabular}

Reverse Parking: The path was divided into 3 blocks consisting Red, yellow and green color. These blocks guide the driver by indicating whether it is safe or he needs to slow down or stop. Whenever an object is detected, it is bounded by a rectangle. If the bottom left point lies inside any block, the whole object is considered to be lying inside the block and hence the driver is alerted. If the object lies in the yellow region, the driver is alerted with slow beep sound and if the object lies in the red region, he is alerted through a strong message (Yang et. al., 2014). An attempt was made to provide Bird's eye view by stitching the videos from 4 Cameras fitted around Car. The real time results have achieved driver satisfaction which was tested in 20 different cars with 50 different drivers.

Pedestrian and Car Detection: The program detects pedestrians of different heights and postures who are 
vulnerable to hit the vehicle. The detection of pedestrian with different height and postures was achieved by training the HAAR classifier using different postures and also images of people with different height. For detecting the pedestrian vulnerable to hit the vehicle, we considered a region of interest consisting only the road and not the entire frame. This also helps in making the program faster as the HAAR classifier has to scan through only a small region instead of the entire frame (Seo et al., 2014). Figure 2 shows the results obtained on all the modules.
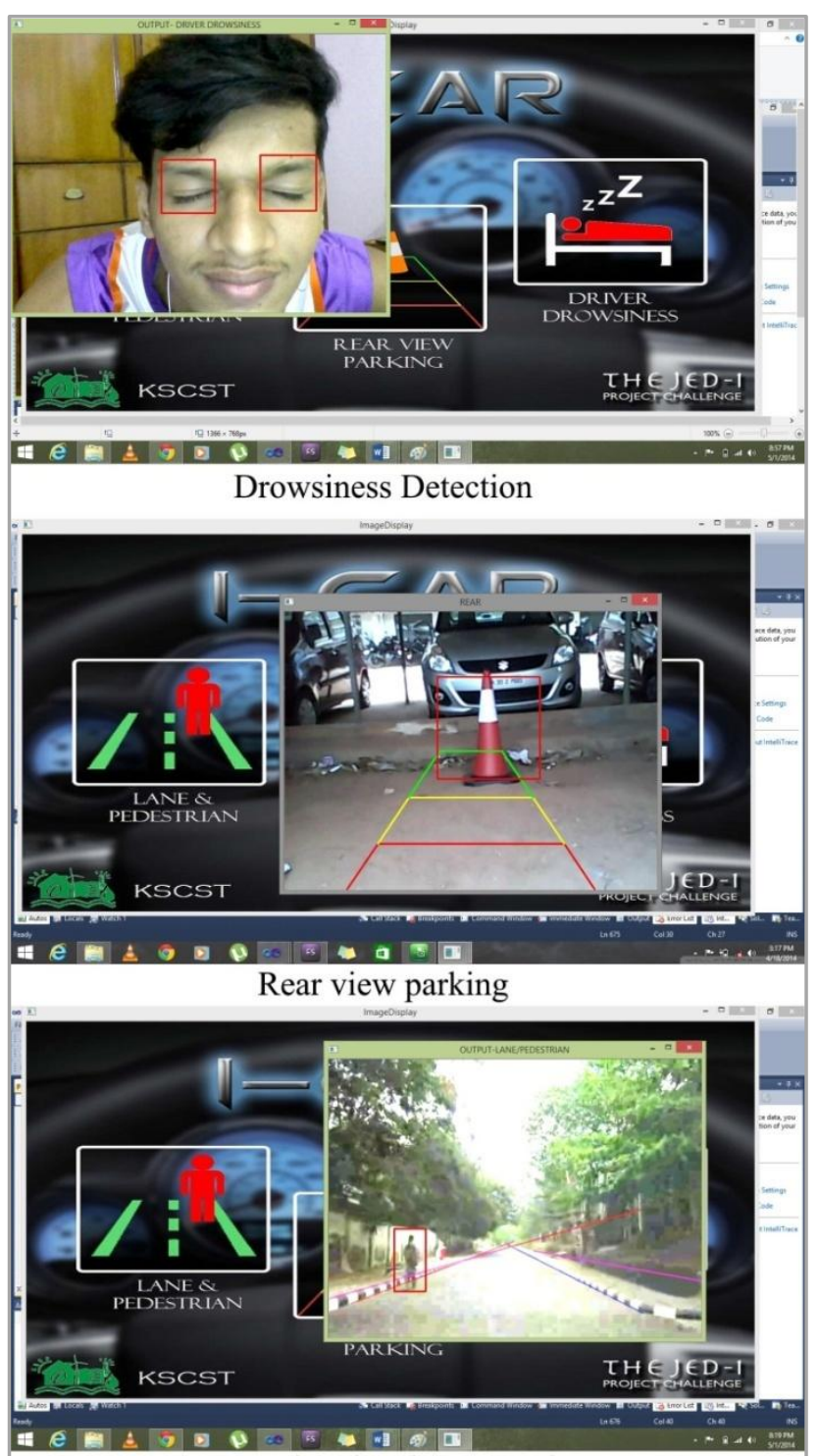

Lane and Pedestrian Detection

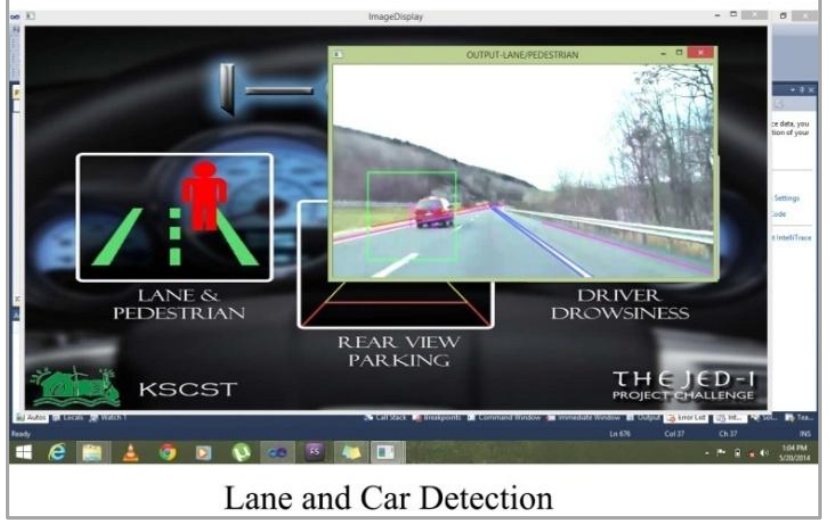

Figure 1: Results of all modules

\section{CONCLUSIONS}

It has been successfully demonstrated that the idea of mediate control takes burden off from the driver and assists him in safe driving. i-Car detects obstacles and prevents collisions without totally removing control from the operator. It also implements techniques such as lane detection to proceed in the right path which is most critical during traffic congestion. Image processing offers a noninvasive approach to detect drowsiness without the annoyance or interference. Even though there is overhead of extra hardware, the system is proved to be most effective in terms of avoiding the accidents that are caused due to human errors.

The present research focuses on alerting or guiding the driver to take necessary actions. The future work may include fully automated system which will respond to these events by itself irrespective of the actions taken by the driver.

\section{REFERENCES}

Prioletti, A., Mogelmose, A., Grisleri, P., Trivedi, M.M., Broggi, A. and Moeslund, T.B. (2013). Part Based Pedestrian Detection and Feature Based Tracking for Driver Assistance: Real Time, Robust Algorithms and Evaluation. Intelligent Transportation System 14(3): 1-14.

Aly Mohamed (2008). Real time Detection of Lane Markers in Urban Streets, IEEE Intelligent Vehicles Symposium, Eindhoven, The Netherlands, June 2008.

Chen, C., Zhang, B., and Gao, S. (2014). A Lane Detection Algorithm Based on Hyperbola Model. In: Computer Engineering and Networking. Lecture Notes in Electrical Engineering Volume 277, pp 609-616.

Government of India, Ministry of Road Transport and Highways (2012). Road Accidents in India, Transport Research Wing.

Hillel, A.B., Lerner, R., Levi, D., and Raz, G. (2014). Recent progress in road and lane detection: a survey. Machine Vision and Applications 25(3): 727-745.

Garcia, I., Bronte, S., Bergasa, L.M., Almazan, J. and Yebes, J. (2012). Vision based drowsiness detector for real driving conditions. Intelligent Vehicles Symposium, pp. 618-623.

Rezaee, K., Alavi, S.R., Madani, M., Rasegh, G.M., Khavari, H. and Haddadnia, J. (2013). Real time intelligent alram system of driver fatigue based on video sequences. Robotics and Mechatronics pp. 378-383 (ISBN: 978-14673-5809-5).

Viola, P., Jones, M., and Snow, D. (2003). Detecting Pedestrians Using Patterns of Motion and Appearance. Mitsubishi Electric Research Laboratories.

Qingquan Li., Long Chen., Ming Li., Shih-Lung Shaw., Nuchter, A., (2014). A Sensor-Fusion Drivable-Region and Lane Detection System for Autonomous Vehicle Navigation in Challenging Road Scenarios. IEEE Transactions on Vehicular Technology 63(2): 540-555.

Seo, S.U., Cheon, S.H. and Jang, S.W. (2014). Design and Implementation of an Around View Image Storing System Based on Car PC. In: Ubiquitous Information Technologies and Applications, pp. 509-517).

Sivaraman, S. and Trivedi, M.M. (2014). Active learning for on-road vehicle detection: A comparative study. Machine VIsion and Applications 25(3): 599-611.

Yang, S.H., Hong, G.S., Ryong, B. and Kim, B.G. (2014). Novel Real-Time Automobile Detection Algorithm for Blind Spot Area. In: Frontier and Innovation in Future Computing and Communications 301: 821-832. 